\title{
Inhomogeneous freeze-out in relativistic heavy ion collisions
}

\author{
Detlef Zschiesche* Instituto de Física, Universidade Federal do Rio de Janeiro \\ C.P. 68528, Rio de Janeiro, RJ 21941-972, Brazil \\ E-mail: detlef@if.ufrj.br
}

\begin{abstract}
The decoupling surface in relativistic heavy-ion collisions may not be homogeneous. Rather, inhomogeneities should form when a rapid transition from high to low entropy density occurs. We analyze the hadron "chemistry" from high-energy heavy-ion reactions for the presence of such density inhomogeneities. We show that due to the non-linear dependence of the particle densities on the temperature and baryon-chemical potential such inhomogeneities should be visible even in the integrated, inclusive abundances. We analyze experimental data from $\mathrm{Pb}+\mathrm{Pb}$ collisions at CERN-SPS and $\mathrm{Au}+\mathrm{Au}$ collisions at BNL-RHIC to determine the amplitude of inhomogeneities and the role of local and global strangeness neutrality.
\end{abstract}

Critical Point and Onset of Deconfinement

July 3-6 2006

Florence, Italy

\footnotetext{
* Speaker.

${ }^{\dagger}$ Work done in collaboration with Adrian Dumitru and Licinio Portugal
} 


\section{Introduction}

It is expected that at sufficiently high energies, a transient state of deconfined matter with broken $Z(3)$ center symmetry and/or with (approximately) restored chiral symmetry is produced in collisions of heavy nuclei. Lattice QCD simulations [2] indicate that a second-order critical point exists, which was predicted by effective chiral Lagrangians [3]; present estimates locate it at $T \approx 160 \mathrm{MeV}, \mu_{B} \approx 360 \mathrm{MeV}$. This point, where the $\sigma$-field is massless, is commonly assumed to be the endpoint of a line of first-order phase transitions in the $\left(\mu_{B}, T\right)$ plane. To detect that endpoint, it is hoped that by varying the beam energy, for example, one can "switch" between the regimes of first-order phase transition and cross over, respectively. If the particles decouple shortly after the expansion trajectory crosses the line of first order transitions one may expect a rather inhomogeneous (energy-) density distribution on the freeze-out surface [ 4 . 河 (similar, say, to the CMB photon decoupling surface observed by WMAP [6]). On the other hand, if the lowtemperature and high-temperature regimes are smoothly connected, pressure gradients tend to wash out density inhomogeneities. Similarly, in the absence of phase-transition induced non-equilibrium effects, the predicted initial-state density inhomogeneities [7, 8] should be strongly damped.

Thus, we investigate the properties of an inhomogeneous fireball at (chemical) decoupling. Note that if the scale of these inhomogeneities is much smaller than the decoupling volume then they can not be resolved individually, nor will they give rise to large event-by-event fluctuations. Because of the nonlinear dependence of the hadron densities on $T$ and $\mu_{B}$, they should nevertheless reflect in the average abundances.

Our basic assumption is that as the fireball expands and cools, at some stage the abundances of hadrons "freeze", keeping memory of the last instant of chemical equilibrium. This stage is refered to as chemical freeze-out. By definition, only processes that conserve particle number for each species individually, or decays of unstable particles may occur later on. The simplest model is to treat the gas of hadrons within the grand canonical ensemble, assuming a homogeneous decoupling volume. The abundances are then determined by two parameters, the temperature $T$ and the baryonic chemical potential $\mu_{B}$; the chemical potential for strangeness is fixed by the condition for overall strangeness neutrality. Fits of hadronic ratios were performed extensively [9, 10] within this model, sometimes also including a strangeness $\left(\gamma_{s}\right)$ or light quark $\left(\gamma_{q}\right)$ supression factor [11, 12] or interactions with the chiral condensate [13].

In [14] we analyzed the experimental data on relative abundances of hadrons with respect to the presence of inhomogeneities on the decoupling surface. To that end we proposed a very simple and rather schematic extension of the common grand canonical freeze-out model, i.e. a superposition of such ensembles with different temperatures and baryon-chemical potentials. Each ensemble is supposed to describe the local freeze-out on the scale of the correlation length $\sim 1 / T \sim 1-2 \mathrm{fm}$. Even if freeze-out occurs near the critical point, the correlation length of the chiral condensate is bound from above by finite size and finite time effects, effectively resulting in similar numbers [15]. On the other hand, for small chemical potential, far from the region where the $\sigma$-field is critical, the relevant scale might be set by the correlation length for Polyakov loops, which is of comparable magnitude [16]. Classical nucleation theory for strong first-order phase transitions predicts even larger "bubbles" [17] but is unlikely to apply to small, rapidly expanding systems encountered in heavy-ion collisions [5, 18]. Another (classical) model for the formation of small droplets in 
rapidly expanding QCD matter has been introduced in [19]. The entire decoupling surface contains many such "domains", even if a cut on mid-rapidity is performed. We therefore expect that the distributions of temperature and chemical potential are approximately Gaussian [20]. Besides simplicity, another goal of the present analysis is to avoid reference to a particular dynamical model for the formation or for the distribution of density perturbations. In fact, we presently aim merely at checking whether any statistically significant signal for the presence of inhomogeneities is found in the data. If so, more sophisticated dynamical models could be employed in the future to understand the evolution of inhomogeneities from their possible formation in a phase transition until decoupling.

Rate equations for nuclear fusion and dissociation processes (and neutron diffusion) have been used for inhomogeneous big bang nucleosynthesis in the early universe [21]. Similarly, hadronic cascade models could be used for heavy-ion reactions [22]. This would remove reference to the grand-canonical ensemble and to a thin decoupling surface in space-time. In fact, hadronic binary rescattering models do predict a rather thick freeze-out layer [22, 23], where matter expands nonideally. On the other hand, the steep drop of multi-particle collision rates with temperature should narrow the freeze-out again [24]. In either case, we do not expect a strong energy dependence of the width of freeze-out (see also [25]).

At chemical freeze-out, matter is in a state of expansion. However, such flow effects do not affect the relative abundances of the particles (in full phase space) if their densities are homogeneous throughout the decoupling volume. The total number of particles of species $i$, integrated over a solid angle of $4 \pi$, is given by an integral of the current $N_{i}^{\mu}=\rho_{i} u^{\mu}$, with $u^{\mu}$ the four-velocity of the expanding fluid, over a given freeze-out hypersurface $\sigma^{\mu}=\left(t^{\mathrm{fo}}, \vec{x}^{\mathrm{fo}}\right)$ :

$$
N_{i}=\int d \sigma_{\mu} N_{i}^{\mu}=\rho_{i}\left(T^{\mathrm{fo}}, \mu_{B}^{\mathrm{fo}}\right) \int u^{\mu} d \sigma_{\mu} .
$$

The second factor on the r.h.s. is nothing but the three-volume $V_{3}$ of the decoupling hypersurface as seen by the observer. This volume is common to all species and drops out of multiplicity ratios: $N_{i} / N_{j}=\rho_{i}^{\mathrm{fo}} / \rho_{j}^{\mathrm{fo}}$. It is clear that the argument holds even when cuts in momentum space are performed, provided that the differential distributions of all particles do not depend on that particular momentum-space variable (for example, rapidity cuts for boostinvariant expansion [26]).

When the intensive variables $T$ and $\mu_{B}$ vary, then the integration measure $\left(\int u \cdot d \sigma\right) / V_{3}$ will, in general, depend on the assumed distribution and amplitude of inhomogeneities, as well as on the hydrodynamic flow profile etc. Nevertheless, it is still the same for all particle species and so can be written in the form

$$
\frac{1}{V_{3}} \int u \cdot d \sigma \longrightarrow \int d T d \mu_{B} P\left(T, \mu_{B}\right)
$$

with $P\left(T, \mu_{B}\right)$ some distribution for $T$ and $\mu_{B}$. For simplicity, and for lack of an obvious motivation for assuming otherwise, we shall take $P\left(T, \mu_{B}\right)$ to factorize into a distribution for $T$, times one for $\mu_{B}$. These distributions could, in principle, be obtained from the real-time evolution of the phase transition [曰, 可].

\section{The model}

In [14] we introduced our model to analyze the available data from heavy-ion collisions at CERN-SPS and BNL-RHIC. There the hadron abundances are determined by four parameters: the 
arithmetic means of the temperatures and chemical potentials of all domains, $\bar{T}$ and $\bar{\mu}_{B}$, and the widths of their Gaussian distributions, $\delta T$ and $\delta \mu_{B}$. Of course, the densities of strange particles depend also on the strangeness-chemical potential $\mu_{S}$, which we determined in [14] by imposing local strangeness neutrality. That means, the strange chemical potential in each single domain was fixed by demanding zero net strangeness there. However, the effect of independent fluctuations of $\mu_{S}$ should also be looked at, in particular for collisions at low and intermediate energies $\left(\sqrt{ } s_{N N} \lesssim 15 \mathrm{GeV}\right)$. This may help for example to reproduce the $\bar{\Lambda}$ to $\bar{p}$ ratio, which was found to be larger than one [28] and the $K^{+} / \pi^{+}$enhancement around $E_{\mathrm{Lab}} / A=30 \mathrm{GeV}$ [29]. Allowing for such independent fluctuations, the hadron abundances depend on six parameters: the arithmetic means of the temperatures and chemical potentials of all domains, $\bar{T}, \bar{\mu}_{B}$ and $\bar{\mu}_{S}$, and the widths of their Gaussian distributions, $\delta T, \delta \mu_{B}$ and $\delta \mu_{S}$. They read:

$$
\begin{aligned}
& \bar{\rho}_{i}\left(\bar{T}, \bar{\mu}_{B}, \bar{\mu}_{S}, \delta T, \delta \mu_{B}, \delta \mu_{S}\right)=\int_{0}^{\infty} d T P(T ; \bar{T}, \delta T) \\
& \int_{-\infty}^{\infty} d \mu_{B} P\left(\mu_{B} ; \bar{\mu}_{B}, \delta \mu_{B}\right) \int_{-\infty}^{\infty} d \mu_{S} P\left(\mu_{S} ; \bar{\mu}_{S}, \delta \mu_{S}\right) \rho_{i}\left(T, \mu_{B}, \mu_{S}\right),
\end{aligned}
$$

with $\rho_{i}\left(T, \mu_{B}, \mu_{S}\right)$ the actual "local" density of species $i$, and with $P(x ; \bar{x}, \delta x) \sim \exp \left[-(x-\bar{x})^{2} / 2 \delta x^{2}\right]$ the distribution of temperatures and chemical potentials within the decoupling three-volume (the proportionality constants normalize the distributions over the intervals where they are defined). In addition, strangeness conservation enters now as a global constraint for the mean of the strange chemical potential $\bar{\mu}_{S}$ :

$$
f_{s}=\sum_{i} \bar{\rho}_{i}\left(\bar{T}, \bar{\mu}_{B}, \bar{\mu}_{S}, \delta T, \delta \mu_{B}, \delta \mu_{S}\right)\left(n_{s}^{i}-n_{\bar{s}}^{i}\right)=0,
$$

with $f_{s}$ the net-strangeness, $n_{s}^{i}, n_{\bar{s}}^{i}$ the number of strange and anti-strange quarks of hadron species $i$, respectively. That means, the global densities obtained for given values of temperature and chemical potential parameters weighted with the corresponding net number of strange quarks are summed and demanded to vanish to guarantee strangeness neutrality. In the limit $\delta T, \delta \mu_{B}, \delta \mu_{S} \rightarrow 0$ the Gaussian distributions are replaced by $\delta$-functions and the conventional homogeneous freezeout scenario is recovered:

$$
\bar{\rho}_{i}\left(\bar{T}, \bar{\mu}_{B}, \bar{\mu}_{S}, 0,0,0\right)=\rho_{i}\left(\bar{T}, \bar{\mu}_{B}, \bar{\mu}_{S}\right),
$$

and the corresponding strangeness neutrality condition fixing $\bar{\mu}_{S}$. In other words, in that limit the average densities are uniquely determined by the first moments of $T$ and $\mu_{B}$. For the present investigation, we set the width of the distribution for the strange chemical potential equal to zero, $\delta \mu_{S}=0$. Since eq.(2.2) only ensures global strangeness neutrality, in this limit still finite net strangeness values in individual domains will appear, in contrast to our former analysis, where we fixed $\mu_{S}$ by $f_{s}=0$ locally. It is important to note that with setting $\delta \mu_{S}=0$ and the global constraint eq. 2.2 for $\bar{\mu}_{S}$, the densities again are a function of four parameters: $\bar{T}, \bar{\mu}_{B}, \delta T$ and $\delta \mu_{B}$. Thus we will write all quantities again as a function of these four parameters only. In the following we will investigate how the fits to the experimentally measured particle abundances are influenced by the different strangeness neutraliy conditions [31]. 
For the present analysis we compute the densities $\rho_{i}\left(T, \mu_{B}\right)$ in the ideal gas approximation, supplemented by an "excluded volume" correction:

$$
\rho_{i}\left(T, \mu_{B}\right)=\frac{\rho_{i}^{\mathrm{id}-\mathrm{gas}}\left(T, \mu_{B}\right)}{1+v_{i} \sum_{j} \rho_{j}^{\mathrm{id}-\mathrm{gas}}} .
$$

This schematic correction models repulsive interactions among the hadrons at high densities. $v_{i}$ denotes the volume occupied by a hadron of species $i$; we employ $v=\frac{4}{3} \pi R_{0}{ }^{3}$ with $R_{0}=0.3 \mathrm{fm}$ for all species [27]. Therefore, for the homogeneous model the denominator in (2.4) drops out of multiplicity ratios. This is not the case for an inhomogeneous decoupling surface, where the distributions of various species differ. For all fits over the full solid angle, we fixed the isospin chemical potential by equating the total charge in the initial and final states; for the mid-rapidity fits at high energies, we fixed $\mu_{I}=0$.

To illustrate the effect of inhomogeneities on the distributions of various hadrons within the decoupling volume we introduce

$$
\begin{aligned}
& D_{i}\left(T ; \bar{T}, \bar{\mu}_{B}, \delta T, \delta \mu_{B}\right)=P(T ; \bar{T}, \delta T) \times \frac{\int_{-\infty}^{\infty} d \mu_{B} P\left(\mu_{B} ; \bar{\mu}_{B}, \delta \mu_{B}\right) \rho_{i}\left(T, \mu_{B}\right)}{\bar{\rho}_{i}\left(\bar{T}, \bar{\mu}_{B}, \delta T, \delta \mu_{B}\right)}, \\
& D_{i}\left(\mu_{B} ; \bar{T}, \bar{\mu}_{B}, \delta T, \delta \mu_{B}\right)=P\left(\mu_{B} ; \bar{\mu}_{B}, \delta \mu_{B}\right) \times \frac{\int_{0}^{\infty} d T P(T ; \bar{T}, \delta T) \rho_{i}\left(T, \mu_{B}\right)}{\bar{\rho}_{i}\left(\bar{T}, \bar{\mu}_{B}, \delta T, \delta \mu_{B}\right)} .
\end{aligned}
$$

$D_{i}(T)$, for example, is the probability that a particle of type $i$ was emitted from a domain of temperature $T$. The main contribution to the integrals in (2.1) is not from $\bar{T}$ and $\bar{\mu}_{B}$ since hot spots shine brighter than "voids". Rather, they are dominated by the stationary points of the distributions defined in eqs. (2.5.2.6) above. Hence, the average emission temperature $\langle T\rangle_{i}$ and baryon-chemical potential $\left\langle\mu_{B}\right\rangle_{i}$ in general depend on the particle species $i$, unless $\delta T=\delta \mu_{B}=0$. They can be evaluated as

$$
\begin{gathered}
\langle T\rangle_{i}=\int_{0}^{\infty} d T T D_{i}\left(T ; \bar{T}, \bar{\mu}_{B}, \delta T, \delta \mu_{B}\right), \\
\left\langle\mu_{B}\right\rangle_{i}=\int_{-\infty}^{\infty} d \mu_{B} \mu_{B} D_{i}\left(\mu_{B} ; \bar{T}, \bar{\mu}_{B}, \delta T, \delta \mu_{B}\right) .
\end{gathered}
$$

Physically, this means that for non-zero widths of the temperature and chemical potential distributions the freeze-out volume is not perfectly "stirred", in that the relative concentrations of the particles vary.

\section{Data analysis}

To determine the four parameters of the model we minimize

$$
\chi^{2}=\sum_{i}\left(r_{i}^{\text {exp }}-r_{i}^{\text {model }}\right)^{2} / \sigma_{i}^{2}
$$


in the space of $\bar{T}, \bar{\mu}_{B}, \delta T$, and $\delta \mu_{B}$. That is, we obtain least-square estimates for the parameters, assuming that they are independent. In (3.1), $r_{i}^{e x p}$ and $r_{i}^{\text {model }}$ denote the experimentally measured and the calculated particle ratios, respectively, and $\sigma_{i}^{2}$ is set by the uncertainty of the measurement. Wherever available, we sum systematic and statistical errors in quadrature.

The data used in our analysis are the particle multiplicities measured by the NA49 collaboration for central $\mathrm{Pb}+\mathrm{Pb}$ collisions at beam energy $E_{\mathrm{Lab}} / A=20,30,40,80$ and $158 \mathrm{GeV}$ [29], and those measured by STAR for central $\mathrm{Au}+\mathrm{Au}$ collisions at BNL-RHIC, ref. [30] ( $\sqrt{ } s_{N N}=130 \mathrm{GeV}$, compiled in [32]) and ref. [33] (200 GeV). At RHIC energies, we analyze the midrapidity data; at top SPS energy, both, midrapidity and $4 \pi$ data. At all other energies, we restrict ourselves to the $4 \pi$ solid angle data by NA49 in order to avoid biases arising from differing acceptance windows of various experiments. Furthermore, our checks showed that the fit results can depend somewhat on the actual selection of experimental ratios. Hence, where possible, we have opted for the least bias by choosing $r_{i}^{e x p}=N_{i}^{e x p} / N_{\pi}^{e x p}$, i.e. the multiplicity of species $i$ relative to that of pions. This represents the maximal set of independent data points, as it is equivalent to fitting absolute multiplicities with an additional overall three-volume parameter, $N_{i}=V_{3} \rho_{i}$.

Specifically, at $E_{\mathrm{Lab}} / A=20,30$, and $80 \mathrm{GeV}$ the multiplicities of $\pi^{+}, \pi^{-}, K^{+}, K^{-}, B-\bar{B}$, $\Lambda, \bar{\Lambda}$, and $\phi$ are available. For the (in-)homogeneous model, this leaves five (three) degrees of freedom. At $40 \mathrm{GeV}$, we can add the $\Xi^{-}$and $\Omega+\bar{\Omega}$. The data sets for top SPS energies include yet a few more species: $p, \bar{p}$ (only midrapidity), $K_{S}^{0}$ (only $4 \pi$ ), $\bar{\Xi}^{+}$and $\Omega, \bar{\Omega}$ seperately. For RHIC-130, we fitted to the $K^{+} / K^{-}, \bar{p} / p, \bar{\Lambda} / \Lambda, \Xi^{+} / \Xi^{-}, \bar{\Omega} / \Omega, K^{-} / \pi^{-}, K_{S}^{0} / \pi^{-}, \bar{p} / \pi^{-}, \Lambda / \pi^{-}, K_{0}^{*} / \pi^{-}, \phi / \pi^{-}$, $\Xi^{-} / \pi^{-}$and $\Omega / \pi^{-}$ratios. Finally, at RHIC-200 the $K^{+} / K^{-}, \bar{p} / p, \bar{\Omega} / \Omega, K^{-} / \pi^{-}, \bar{p} / \pi^{-}, \Lambda / \pi^{-}$, $\bar{\Lambda} / \pi^{-}, \Xi^{-} / \pi^{-}, \Xi^{+} / \pi^{-}, \Omega / \pi^{-}, \phi / K^{-}$and $K_{0}^{*} / K^{-}$ratios were used. The first three ratios are close to unity and essentially just set the chemical potentials to zero; they do not help to fix $\bar{T}, \delta T$ and $\delta \mu_{B}$.

Where appropriate, feeding from strong and electromagnetic decays has been included in $r_{i}^{\text {model }}$ by replacing $\rho_{i} \rightarrow \rho_{i}+B_{i j} \rho_{j}$. The implicit sum over $j \neq i$ runs over all unstable hadron species, with $B_{i j}$ the branching ratio for the decay $j \rightarrow i$, which were taken from [34]. From all the resonances listed by the Particle Data Group [34], mesons up to a mass of $1.5 \mathrm{GeV}$ and baryons up to a mass of $2 \mathrm{GeV}$ were included, respectively. The finite widths of the resonances were not taken into account, and unknown branching ratios were excluded from the feeding. These details are irrelevant for the qualitative behavior of $\delta T$ and $\delta \mu_{B}$ but do, of course, matter for quantitative results.

\section{Results}

Fig. 11 shows the minimal $\chi^{2}$ per degree of freedom (taken as the number of data points minus the number of parameters) for the homogeneous approach and the inhomogeneous approach with local or global strangeness neutrality, respectively. Note that the $\chi^{2}$-values for the homogeneous model are in general agreement with the analysis done in [9] and other data from the literature [10, 11, 33]. As already shown in [14] and in general accordance with the analysis done in [9], for intermediate SPS energies, $E_{\mathrm{Lab}} / A \simeq 30-160 \mathrm{GeV}, \chi^{2} /$ dof is considerably smaller for the inhomogeneous freeze-out surface than for the homogeneous case, which is far outside the 95.4\% confidence interval [35]. At $E_{\mathrm{Lab}} / A=20 \mathrm{GeV}$ and at RHIC energies, $\chi^{2} /$ dof is similar 


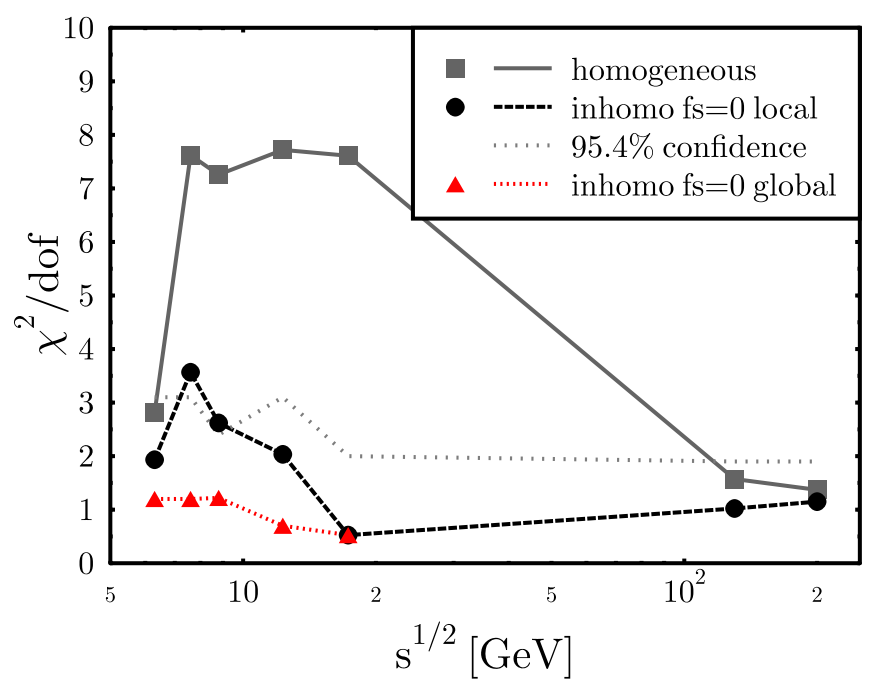

Figure 1: $\chi^{2} /$ dof versus $\sqrt{ } s_{N N}$ for the homogeneous ( $\delta T=\delta \mu=0$, squares) and the inhomogeneous fit ( $\delta T$ and $\delta \mu$ free parameters, circles and triangles). Circles denote the case of local strangeness neutrality, while triangles represent the global strangeness neutrality case. The lines are meant to guide the eye. Furthermore, the $\chi^{2} /$ dof corresponding to the $95.4 \%$ confidence interval is shown by the dotted line.

for the inhomogeneous approach with local strangeness neutrality and the homogenous model. However, between 20 and $80 \mathrm{GeV}$ the $\chi^{2} /$ dof values for the inhomogenous approach with local strangeness neutrality are rather large (between 2 and 4). In contrast, the inhomogenoues model with global strangeness neutrality gives $\chi^{2} / d o f \approx 1$ for $E_{\mathrm{Lab}} / A \simeq 20-160 \mathrm{GeV}$. It is important to note that this result is not due to introducing an additional parameter, but just due to allowing for domains of finite strangeness with global strangeness neutrality! The calculations using global strangeness conservation for RHIC energies are under way, but due to the corresponding small baryon chemical potentials at these high energies no considerable effect should be expected. Thus, the inhomogenoues model allowing for domains of finite net strangeness gives a very satisfactory description $\left(\chi^{2} / d o f \approx 1\right)$ of the experimental data for particle abundance ratios from lowest SPS energies up to highest RHIC energies. However, at RHIC the homogeneous approach already gives a good description of the data and the inhomogeneous model does not provide a statistically significant improvement. Thus, the assumption of a nearly homogeneous decoupling surface can not be rejected there. On the other hand, the considerable improvement of the description of the data for $E_{\mathrm{Lab}} / A \simeq 30-160 \mathrm{GeV}$ indicates that at intermediate and high SPS energies, the experimental data favor an inhomogeneous freeze-out surface. For the SPS $20 \mathrm{GeV}$ data the situation is not clear: there is certainly a reduction of the $\chi^{2} / d o f$ in the inhomogeneous approach, but also the homogeneous model gives a much better value than for higher SPS energies. Here more experimental data are necessary to clarify the picture. It is worth noting that in general, the improvement due to the inhomogenoues decoupling surface is not driven by one single species; rather, the inhomogeneous model describes nearly all multiplicities better than a homogeneous decoupling surface [36].

To illustrate the significance of inhomogeneities differently, we show contours of $\chi^{2} /$ dof in the plane of $\delta T, \delta \mu_{B}$ in figs.2, 3, and $甘$. Here, $\bar{T}$ and $\bar{\mu}_{B}$ were allowed to vary freely such as to minimize $\chi^{2}$ at each point. Fig. 目 shows that at RHIC energy, $\chi^{2}$ is very flat in both directions. 
This shows again that with the present data points, a homogeneous freeze-out model appears to be a reasonable approximation at high energies. In contrast, fig. 3 shows that $\chi^{2}$ is relatively flat along

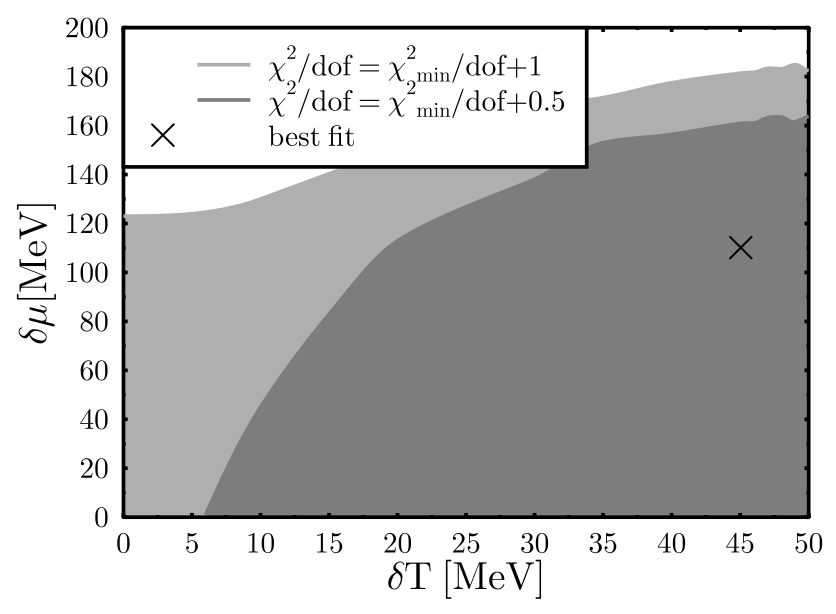

Figure 2: $\chi^{2} /$ dof contours in the $\delta T, \delta \mu_{B}$ plane for top RHIC energy, $\left(\sqrt{ } s_{N N}=200 \mathrm{GeV}\right)$. The other two parameters $\left(\bar{T}, \bar{\mu}_{B}\right)$ are allowed to vary freely. The $\chi^{2} /$ dof minimum is indicated by the cross.

the $\delta \mu_{B}$ direction, while $\delta T$ is determined more accurately and is clearly non-zero. In general we find that in the approach with local strangeness neutrality there is little correlation between $\delta T$ and $\delta \mu_{B}$ and that about the minimum, $\chi^{2}$ is rather flat in $\delta \mu_{B}$ direction for all energies. Finally, fig. 3

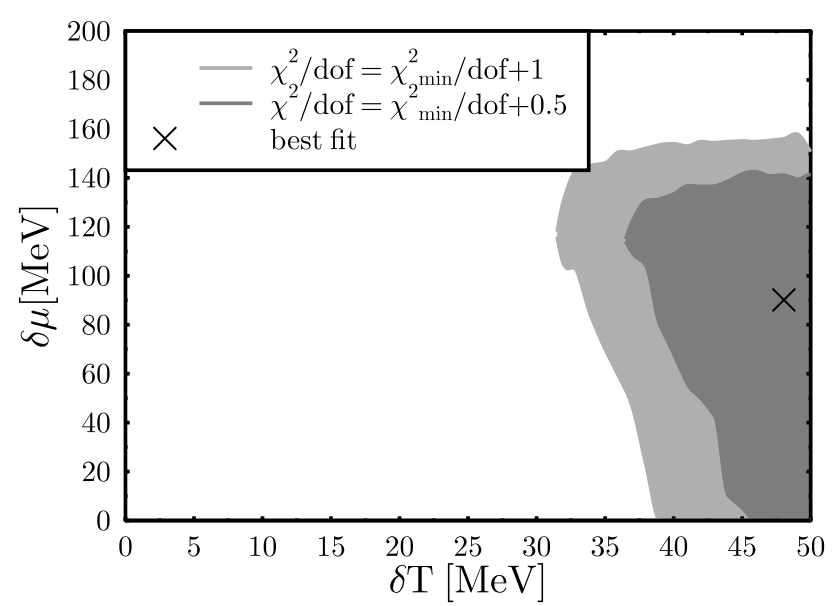

Figure 3: Same as fig. 2 for top SPS energy $\left(E_{\mathrm{Lab}}=158 \mathrm{GeV}\right)$ with local strangeness neutrality.

shows the contours at SPS 158 for the case of global strangeness neutrality. Now, the $\chi^{2}$ determines the $\delta \mu_{B}$ more accurately, favoring relatively large finite values. For $\delta T$, again, values different from zero are strongly favored, which, however, turn out to be generally a little bit smaller than in the local $f_{s}=0$ case. The better description of the data and the better accuracy in determining the width of the $\mu_{B}$-distribution can be explained as follows: If vanishing net strangeness is demanded in each single domain, in regions with high baryon chemical potential the strange chemical potential has to be small to guarantee $f_{s}=0$. Thus, the possible increased production of strange particles in domains with high baryon chemical potential is restricted and results in the shown flatness of the $\chi^{2}$ distribution in $\delta \mu_{B}$ direction. In contrast, if the net strangeness vanishes globally, in domains of 


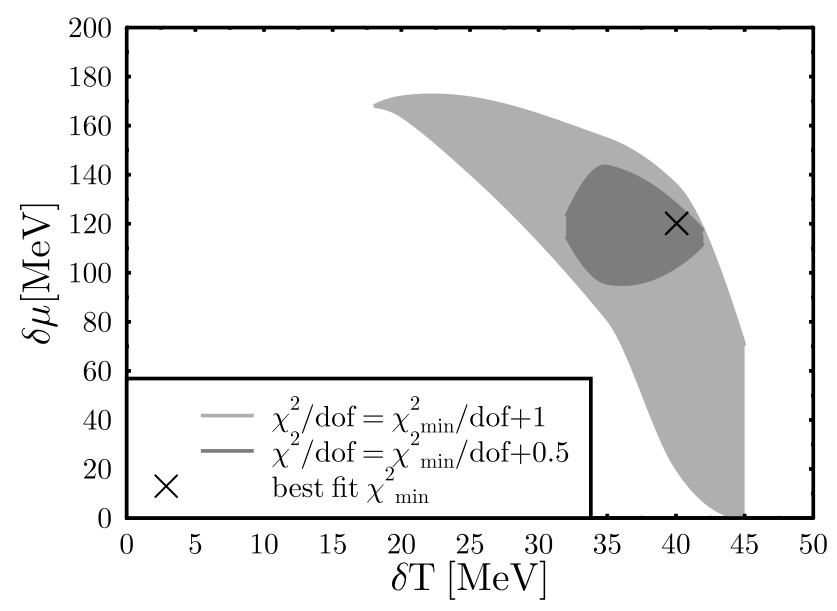

Figure 4: Same as fig. 2 for top SPS energy $\left(E_{\mathrm{Lab}}=158 \mathrm{GeV}\right)$ with global strangeness neutrality.

high chemical potential resulting from a large width $\delta \mu_{B}$, a large number of strange particles can be produced. Thus, the $\chi^{2}$ should be much more sensitive to the value of $\delta \mu_{B}$.

As already discussed in section 2, an inhomogeneous freeze-out surface or finite values for the width-parameters result in different mean emission temperatures and chemical potentials for different particle species, c.f. eq. 2.7. These are shown in fig. 5 for the case of local strangeness neutrality and in fig. 6 for the case of global strangeness neutrality at selected energies in the CERN-SPS range. For the cases shown, the inhomogeneities determined from the fits to the particle abundances are large. Note that the different values for these mean emission temperatures and chemical potentials result from the convolution of the distribution function for a given particle species with the Gaussian probability distribution determined by the four parameters $\bar{T}, \overline{\mu_{B}}, \delta T, \delta \mu_{B}$. For both cases, the effect of the inhomogeneities is evident. For example, anti-protons are typically emitted from regions with lower baryon-chemical potential than protons; also, heavy particles are concentrated in "hot spots" while light pions are distributed more evenly throughout the decoupling volume etc. [37]. Figures 5 and 6 also show the differences in the resulting mean emission temperatures and chemical potentials, depending on whether local or global strangeness neutrality is adopted: In the case of local strangeness neutrality, the emission chemical potentials of baryons and the corresponding anti-baryons do differ much less than in the case of globally vanishing netstrangeness. For example the mean emission baryon chemical potential for the $\Omega$ and the $\bar{\Omega}$ are nearly identical for $f_{s}=0$ locally, while they are widely separated for the global constraint. This results from the above discussed effect of the adjustement of the strange chemical potential in the local case. There, $\mu_{S}$ is so small (large negative value) in regions with large $\mu_{B}$, that the resulting chemical potential of $\Omega$ and $\bar{\Omega}$ are similar. This is not the case anymore if the strange chemical potential is determined globally and constant for the different domains. Then, the mean freeze-out points for the different particle species are spread over a much wider range in the $T-\mu_{B}$-plane. On the other hand, the spread in temperature is somewhat larger in the local case than for global strangeness neutrality, resulting from the larger best fit values for the width parameters $\delta T$. 


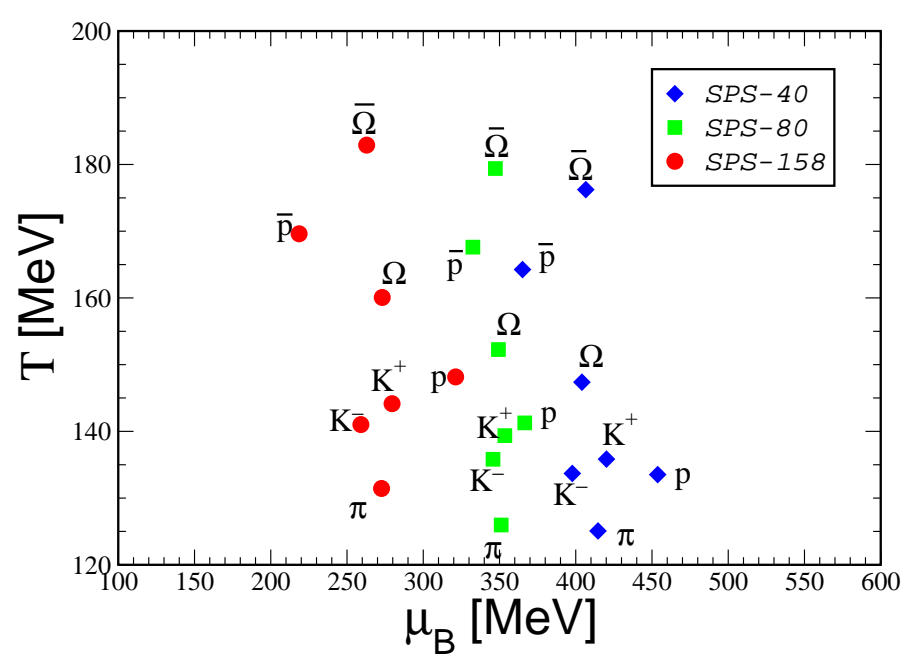

Figure 5: $\quad$ Freeze-out temperatures $\langle T\rangle_{i}$ and chemical potentials $\left\langle\mu_{B}\right\rangle_{i}$ of various particle species at $E_{\mathrm{Lab}} / A=40,80,158 \mathrm{GeV}$ for local strangeness neutrality.

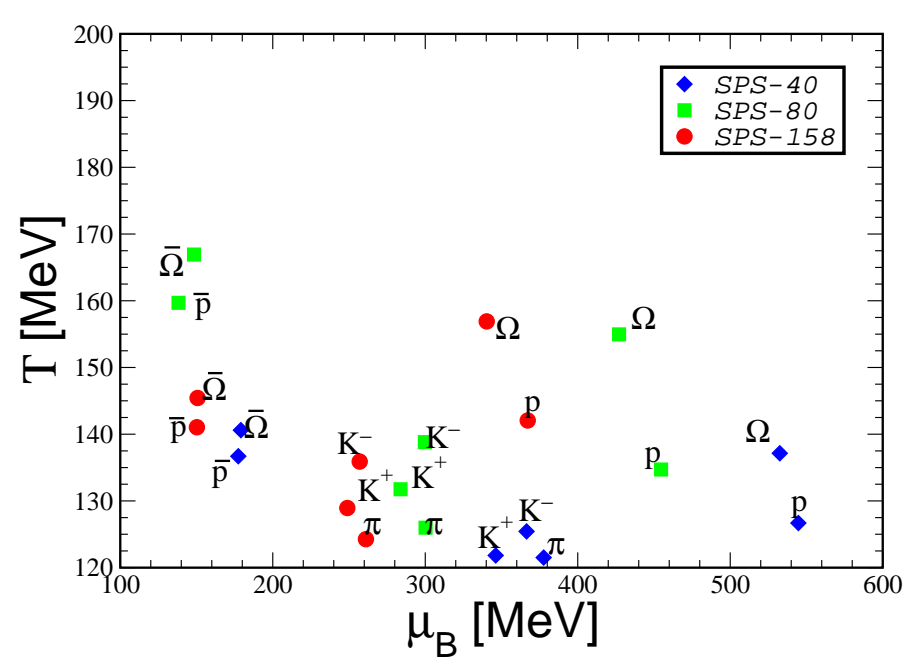

Figure 6: Freeze-out temperatures $\langle T\rangle_{i}$ and chemical potentials $\left\langle\mu_{B}\right\rangle_{i}$ of various particle species at $E_{\mathrm{Lab}} / A=40,80,158 \mathrm{GeV}$ for global strangeness neutrality.

\section{Summary and Outlook}

In summary, we have shown that inhomogeneities on the freeze-out hypersurface do not average out but reflect in the $4 \pi$ (or midrapidity), single-inclusive abundances of various particle species. This is due to the non-linear dependence of the hadron densities $\rho_{i}\left(T, \mu_{B}\right)$ on the local temperature and baryon-chemical potential. Consequently, even the average $\bar{\rho}_{i}$ probe higher moments of the $T$ and $\mu_{B}$ distributions. In [14] we showed that an inhomogenoues freeze-out model with local strangeness conservation strongly improves the description of the data at medium and top 
SPS energies compared to the homogeneous freeze-out. Here we showed that inducing global strangeness neutrality, results, without adding an additional parameter, in a further reduction of the $\chi^{2}$ at SPS energies. With the resulting $\chi / d o f \approx 1$ for the whole range - from lowest SPS to highest RHIC energies. Furthermore, while for local strangeness neutrality we observed a rather flat $\chi^{2} /$ dof in $\delta \mu_{B}$ direction, this is determined more accuratrely if strangeness neutrality is ensured only globally. Rather in this approach a high statistical significance for a finite width of the distributions for temperature and baryon chemical potential at medium and high SPS energies is observed. In addition we showed how in this region the mean emission temperature and chemical potential vary for different particle species. Our results also show that there are some characteristic differences in the distribution of the resulting mean emission values, depending on whether strangeness neutrality is fulfilled locally or globally. Especially the separation in the mean emission chemical potential between baryons and the corresponding anti-baryons is strongly influenced by the adopted strangeness neutrality condition.

Inhomogeneities could also affect the coalescence probabilities of (anti-) nucleons to light (anti-) nuclei, which are also sensitive to density perturbations [39]. Other signals, such as twoparticle correlations [8, 40], could also be analyzed in this regard. Future studies should shed more light on whether these inhomogeneities can indeed be interpreted as fingerprints of a first-order phase transition. Eventually, one would want to establish more quantitative relations between the amplitudes of the $T, \mu_{B}$ inhomogeneities and the properties of the phase transition, e.g. its latent heat and interface tension.

Data from GSI-FAIR, the low energy program at RHIC and and CERN-LHC will provide additional constraints for the evolution of chemical freeze-out with energy.

To improve the quality of the statistical fits, more data on hadron multiplicities would be helpful, in particular at the lower end of the CERN-SPS energy spectrum and at RHIC. This includes estimates of multiplicities of unstable resonances $\left(\rho, K^{*}, \omega, \Delta \ldots\right)$ at chemical freezeout [41]. Data from GSI-FAIR and CERN-LHC will provide additional constraints for the evolution of chemical freeze-out with energy.

\section{Acknowledgements}

I thank C. Greiner for fruitful remarks concerning the model, C. Blume and M. Gazdzicki for helpful discussions about the NA49 data and A. Grunfeld for helping with the construction of the resonance table, CAPES and CNPq for partial support and the organizers of the workshop 'Critical Point and Onset of Deconfinement' July 3-6, 2006, Florence, where this work was presented.

\section{References}

[1] H. Stöcker et al., Nucl. Phys. A 566 (1994) 15c; Nucl. Phys. A 590 (1995) 271c;

[2] Z. Fodor and S. D. Katz, JHEP 0404 (2004) 050.

[3] M. Stephanov, K. Rajagopal and E. V. Shuryak, Phys. Rev. Lett. 81, (1998) 4816.

[4] D. Bower and S. Gavin, Phys. Rev. C 64 (2001) 051902. 
[5] K. Paech and A. Dumitru, Phys. Lett. B 623 (2005) 200; K. Paech, H. Stöcker and A. Dumitru, Phys. Rev. C 68 (2003) 044907; O. Scavenius, A. Dumitru and A. D. Jackson, arXiv:hep-ph/0103219, figs. 5,6 .

[6] http://map.gsfc.nasa.gov/m_mm.html

[7] M. Gyulassy, D. H. Rischke and B. Zhang, Nucl. Phys. A 613 (1997) 397; M. Bleicher et al., Nucl. Phys. A 638 (1998) 391; H. J. Drescher, S. Ostapchenko, T. Pierog and K. Werner, Phys. Rev. C 65 (2002) 054902.

[8] O. J. Socolowski, F. Grassi, Y. Hama and T. Kodama, Phys. Rev. Lett. 93 (2004) 182301.

[9] A. Andronic, P. Braun-Munzinger and J. Stachel, Nucl. Phys. A 772, 167 (2006) [arXiv:nucl-th/0511071].

[10] see for example K. Redlich, J. Cleymans, H. Oeschler and A. Tounsi, Acta Phys. Polon. B 33 (2002) 1609; P. Braun-Munzinger, K. Redlich and J. Stachel, arXiv:nucl-th/0304013; M. Michalec, arXiv:nucl-th/0112044; and references therein.

[11] J. Rafelski, Phys. Lett. B 262 (1991) 333; J. Rafelski, J. Letessier and A. Tounsi, Acta Phys. Polon. B 27 (1996) 1037; F. Becattini, J. Cleymans, A. Keranen, E. Suhonen and K. Redlich, Phys. Rev. C 64 (2001) 024901; F. Becattini, M. Gazdzicki, A. Keranen, J. Manninen and R. Stock, Phys. Rev. C 69 (2004) 024905; G. Torrieri, S. Steinke, W. Broniowski, W. Florkowski, J. Letessier and J. Rafelski, arXiv:nucl-th/0404083; S. Wheaton and J. Cleymans, arXiv:hep-ph/0412031; J. Letessier and J. Rafelski, arXiv:nucl-th/0504028.

[12] F. Becattini, J. Manninen and M. Gazdzicki, arXiv:hep-ph/0511092.

[13] D. Zschiesche, S. Schramm, J. Schaffner-Bielich, H. Stöcker and W. Greiner, Phys. Lett. B 547 (2002) 7; W. Florkowski, W. Broniowski and M. Michalec, Acta Phys. Polon. B 33 (2002) 761; D. Zschiesche, G. Zeeb, K. Paech, H. Stöcker and S. Schramm, J. Phys. G 30 (2004) S381; K. Paech et al., Acta Phys. Hung. A 21, 151 (2004).

[14] A. Dumitru, L. Portugal and D. Zschiesche, Phys. Rev. C 73 (2006) 024902 [arXiv:nucl-th/0511084].

[15] B. Berdnikov and K. Rajagopal, Phys. Rev. D 61 (2000) 105017; K. Paech, Eur. Phys. J. C 33 (2004) S627.

[16] A. Dumitru and R. D. Pisarski, Phys. Lett. B 504 (2001) 282; Nucl. Phys. A 698 (2002) 444.

[17] L. Van Hove, Z. Phys. C 21 (1983) 93; M. Gyulassy, K. Kajantie, H. Kurki-Suonio and L. D. McLerran, Nucl. Phys. B 237 (1984) 477; L. P. Csernai and J. I. Kapusta, Phys. Rev. D 46 (1992) 1379.

[18] O. Scavenius, A. Dumitru, E. S. Fraga, J. T. Lenaghan and A. D. Jackson, Phys. Rev. D 63 (2001) 116003; E. S. Fraga and R. Venugopalan, PhysicaA 345 (2004) 121; Braz. J. Phys. 34 (2004) 315.

[19] I. N. Mishustin, Phys. Rev. Lett. 82 (1999) 4779.

[20] The investigation of other distributions and also the application of the "superstatistics" approach [42] are under way.

[21] see, for example, J. F. Lara, Phys. Rev. D 72 (2005) 023509 for a recent discussion of BBN in an inhomogeneous early universe, and for links to earlier literature.

[22] S. A. Bass et al., Phys. Rev. C 60 (1999) 021902; Phys. Rev. C 61 (2000) 064909; Phys. Lett. B 460 (1999) 411; S. Soff, S. A. Bass and A. Dumitru, Phys. Rev. Lett. 86 (2001) 3981; D. Teaney, J. Lauret and E. V. Shuryak, arXiv:nucl-th/0110037; C. Nonaka and S. A. Bass, arXiv:nucl-th/0510038; T. Hirano, U. W. Heinz, D. Kharzeev, R. Lacey and Y. Nara, arXiv:nucl-th/0511046. 
[23] H. Sorge, Phys. Lett. B 373 (1996) 16.

[24] P. Braun-Munzinger, J. Stachel and C. Wetterich, Phys. Lett. B 596 (2004) 61.

[25] D. Adamova et al. [CERES Collaboration], Phys. Rev. Lett. 90 (2003) 022301.

[26] section II in J. Cleymans and K. Redlich, Phys. Rev. C 60 (1999) 054908; for the general argument for $4 \pi$ ratios see e.g. eqs. (1-3) in M. Bleicher et al., Phys. Rev. C 59 (1999) 1844.

[27] P. Braun-Munzinger, I. Heppe, J. Stachel, Phys. Lett. B 465 (1999) 15.

[28] C. Höhne (for the NA49 collaboration), nucl-ex/0510049. Our present best fit (of the $4 \pi$ ratios) for the inhomogeneous model without independent fluctuations of $\mu_{S}$ yields $\bar{\Lambda} / \bar{p}=0.93$ at $E_{\mathrm{Lab}} / A=40 \mathrm{GeV}$, for example, versus 0.76 for $\delta T=\delta \mu_{B}=0$ (without contributions from weak decays).

[29] M. Gazdzicki et al. [NA49 Collaboration], J. Phys. G 30 (2004) S701 [arXiv:nucl-ex/0403023]; A. Richard [NA49 Collaboration], J. Phys. G 31, S155 (2005); C. Blume [NA49 Collaboration], J. Phys. G 31, S685 (2005) [arXiv:nucl-ex/0411039]; C. Alt et al. [The NA49 Collaboration], J. Phys. G 30, S119 (2004) [arXiv:nucl-ex/0305017]; S. V. Afanasiev et al. [The NA49 Collaboration], Phys. Rev. C 66, 054902 (2002) [arXiv:nucl-ex/0205002]; T. Anticic et al., Phys. Rev. C 69, 024902 (2004); S. V. Afanasiev et al., Nucl. Phys. A 715 (2003) 161 [arXiv:nucl-ex/0208014]; T. Anticic et al. [NA49 Collaboration], Phys. Rev. Lett. 93, 022302 (2004) [arXiv:nucl-ex/0311024]; C. Meurer [NA49 Collaboration], J. Phys. G 30, S1325 (2004) [arXiv:nucl-ex/0406016]; M. Mitrovski [NA49 Collaboration], arXiv:nucl-ex/0406011; C. Alt et al. [NA49 Collaboration], Phys. Rev. Lett. 94, 192301 (2005) [arXiv:nucl-ex/0409004]; S. V. Afanasev et al. [NA49 Collaboration], Phys. Lett. B 491, 59 (2000); A. Mischke et al., Nucl. Phys. A 715, 453 (2003) [arXiv:nucl-ex/0209002]; V. Friese [NA49 Collaboration], Nucl. Phys. A 698, 487 (2002); S. V. Afanasiev et al. [NA49 Collaboration], Phys. Lett. B 538, 275 (2002) [arXiv:hep-ex/0202037]; S. V. Afanasiev et al. [NA49 Collaboration], Phys. Lett. B 538, 275 (2002) [arXiv:hep-ex/0202037].

[30] J. Adams et al. [STAR Collaboration], arXiv:nucl-ex/0311017; M. Calderon de la Barca Sanchez, arXiv:nucl-ex/0111004; C. Adler et al. [STAR Collaboration], Phys. Rev. Lett. 89 (2002) 092301 [arXiv:nucl-ex/0203016]; C. Adler et al. [STAR Collaboration], Phys. Lett. B 595 (2004) 143 [arXiv:nucl-ex/0206008]; C. Adler et al. [STAR Collaboration], Phys. Rev. C 66 (2002) 061901 [arXiv:nucl-ex/0205015]; C. Adler et al. [STAR Collaboration], Phys. Rev. Lett. 87 (2001) 262302 [arXiv:nucl-ex/0110009]; C. Adler et al. [the STAR Collaboration], Phys. Rev. Lett. 86 (2001) 4778 [Erratum-ibid. 90 (2003) 119903] [arXiv:nucl-ex/0104022]; C. Adler et al., Phys. Rev. C 65 (2002) 041901. J. Castillo [STAR Collaboration], Nucl. Phys. A 715 (2003) 518 [arXiv:nucl-ex/0210032]; J. Adams et al. [STAR Collaboration], Phys. Rev. Lett. 92 (2004) 182301 [arXiv:nucl-ex/0307024];

J. Adams et al. [STAR Collaboration], Phys. Lett. B 567 (2003) 167 [arXiv:nucl-ex/0211024];

C. Suire [STAR Collaboration], Nucl. Phys. A 715 (2003) 470 [arXiv:nucl-ex/0211017].

[31] Calculations for finite values of $\delta \mu_{S}=0$ are under way. However, checking the influence of global strangeness conservation without adding an additional parameter represents the first step to be taken in this direction.

[32] J. Cleymans, B. Kämpfer, M. Kaneta, S. Wheaton and N. Xu, Phys. Rev. C 71 (2005) 054901.

[33] O. Barannikova [STAR Collaboration], arXiv:nucl-ex/0403014; J. Adams et al. [STAR Collaboration], arXiv:nucl-ex/0501009; J. Adams et al. [STAR Collaboration], Phys. Rev. Lett. 92 (2004) 112301 [arXiv:nucl-ex/0310004];

[34] S. Eidelman et al. [Particle Data Group Collaboration], Phys. Lett. B 592 (2004) 1. 
[35] For SPS-158 only the $4 \pi$ fit is shown; restricting the homogeneous fit to the mid-rapidity data gives smaller $\chi^{2} / d o f$, but still significantly higher than in the inhomogeneous approach. $\chi^{2}$ is smaller if other particle ratios are considered, as for example $\Xi / \Lambda, \Omega / \Xi$ instead of $\Xi / \pi, \Omega / \pi[9]$ or if finite widths of resonances are taken into account [12]. However, the increase of $\chi^{2}$ at SPS energies is generic if $\mathrm{Na} 494 \pi$-data are fitted.

[36] D. Zschiesche, arXiv:nucl-th/0505054, fig. 7.

[37] Note that the rather high temperatures of the hot spots from which the heavy particles emerge might indicate the need for a better treatment of interactions [13] than the simple excluded-volume model employed here.

[38] E. V. Shuryak and M. A. Stephanov, Phys. Rev. C 63 (2001) 064903; M. Abdel-Aziz and S. Gavin, Phys. Rev. C 70 (2004) 034905.

[39] see e.g. eq. (30) in B. L. Ioffe, I. A. Shushpanov and K. N. Zyablyuk, Int. J. Mod. Phys. E 13 (2004) 1157

[40] H. Heiselberg and A. D. Jackson, arXiv:nucl-th/9809013; S. J. Lindanbaum, R. S. Longacre and M. Kramer, arXiv:nucl-th/0304082; W. N. Zhang, S. X. Li, C. Y. Wong and M. J. Efaaf, Phys. Rev. C 71 (2005) 064908.

[41] C. Markert, J. Phys. G 31 (2005) S169.

[42] C. Beck, Phys. Rev. Lett. 87 (2001) 180601; C. Beck and E. G. D. Cohen, Physica A 322 (2003) 267; H. Touchette and C. Beck, Phys. Rev. E 71 (2005) 016131. 